\title{
Ultra-small superparamagnetic particles of iron oxide in magnetic resonance imaging of cardiovascular disease
}

This article was published in the following Dove Press journal:

Journal of Vascular Diagnostics

3 October 2014

Number of times this article has been viewed

\author{
Colin G Stirrat' \\ Alex T Vesey' \\ Olivia MB McBride' \\ Jennifer MJ Robson' \\ Shirjel R Alam' \\ William A Wallace ${ }^{2}$ \\ Scott I Semple ${ }^{1,3}$ \\ Peter A Henriksen' \\ David E Newby' \\ 'British Heart Foundation Centre for \\ Cardiovascular Science, University \\ of Edinburgh, Edinburgh, UK; \\ ${ }^{2}$ Department of Pathology, University \\ of Edinburgh, Edinburgh, UK; ${ }^{3}$ Clinical \\ Research Imaging Centre, University \\ of Edinburgh, Edinburgh, UK
}

\begin{abstract}
Ultra-small superparamagnetic particles of iron oxide (USPIO) are iron-oxide based contrast agents that enhance and complement in vivo magnetic resonance imaging (MRI) by shortening $\mathrm{T} 1, \mathrm{~T} 2$, and $\mathrm{T} 2 *$ relaxation times. USPIO can be employed to provide immediate blood pool contrast, or to act as subsequent markers of cellular inflammation through uptake by inflammatory cells. They can also be targeted to specific cell-surface markers using antibody or ligand labeling. This review will discuss the application of USPIO contrast in MRI studies of cardiovascular disease.
\end{abstract}

Keywords: cardiac, aortic, MRI, USPIO, carotid, vascular, molecular imaging

\section{Introduction}

Beyond the anatomical and morphological information afforded by standard clinical cardiovascular imaging techniques, it is increasingly recognized that additional information regarding the underlying biological processes involved would be valuable in a range of cardiovascular diseases to aid understanding of disease pathogenesis, provide diagnostic information, predict disease progression, and to monitor response to treatment. Novel molecular and cellular imaging techniques can identify metabolic and biological processes that are critical to disease pathogenesis, and therefore have the potential to predict clinical events and guide therapeutic intervention.

A range of potential imaging modalities exists (Table 1). Magnetic resonance imaging (MRI) has a number of advantages including excellent discrimination of soft tissue components, good spatial resolution and avoidance of ionizing radiation, a particular consideration if serial imaging is envisaged.

Iron oxide particles are a group of MRI contrast agents that can be used for vascular, molecular and cellular imaging. ${ }^{1}$ Iron oxide particles can be categorized according to their size: nano-sized very small superparamagnetic particles of iron oxide (VSPIO, $<20 \mathrm{~nm}$ in diameter), ultra-small superparamagnetic particles of iron oxide (USPIO, 20-50 nm in diameter), small superparamagnetic particles of iron oxide (SPIO, $60-250 \mathrm{~nm}$ in diameter), and micro-sized particles of iron oxide (MPIO, $1-8 \mu \mathrm{m}$ in diameter). VSPIO and USPIO are often classified together (as USPIO) due to similar size. Superparamagnetism occurs when the size of the iron oxide crystal is smaller than that of ferromagnetic domains (approximately $30 \mathrm{~nm}$ ). Under the influence of a strong external magnetic field, such as within an MRI scanner, iron oxide nanoparticles have a large magnetic moment, but unlike larger ferromagnetic particles, this magnetic moment is lost when the field is removed.
Correspondence: Colin G Stirrat British Heart Foundation Centre for Cardiovascular Science, Room SU 305, Chancellor's Building, University of Edinburgh, 49 Little France Crescent, Edinburgh EHI 6 4SB, UK

Tel +44 I3। 24265 I5

Fax +44 I3। 2426379

Email colin.stirrat@ed.ac.uk 
Table I Summary of commonly used molecular imaging modalities

\begin{tabular}{|c|c|c|c|c|c|c|}
\hline Modality & Spatial resolution & Temporal resolution & Sensitivity & Penetration & Radiation & Cost \\
\hline MRI & $10-100$ micron & High & Low & Good & No & High \\
\hline US & 50 micron & High & Medium & $\mathrm{cm}$ & No & Medium \\
\hline SPECT & $\mathrm{I}-2 \mathrm{~mm}$ & Low & Medium & Good & Yes & Medium \\
\hline PET & $\mathrm{I}-2 \mathrm{~mm}$ & Low & High & Good & Yes & High \\
\hline Fluorescence & $2-3 \mathrm{~mm}$ & High & High & $<\mathrm{I} \mathrm{cm}$ & Yes/no & Low \\
\hline
\end{tabular}

Abbreviations: MRI, magnetic resonance imaging; US, ultrasound; SPECT, single-photon emission computerised tomography; PET, positron emission tomography.

USPIO generally consist of an iron oxide core surrounded by a coating, usually of carbohydrate or polymer construction, with a total particle diameter of less than $50 \mathrm{~nm}$. The purpose of the coating is to prevent aggregation, to retain particles within a colloidal suspension and to provide a surface for conjugation of drug molecules and targeting ligands. A range of particles exists varying in size and coating; these two properties are the key determinants of biodistribution, magnetic behavior, and thus the potential applications of the particle., ${ }^{2,3}$ The majority of proposed applications for iron oxide nanoparticles utilize particles within the ultra-small and small size ranges.

Following administration, cells of the reticuloendothelial system including inflammatory macrophages internalize iron oxide nanoparticles. Neutrophils and structural endothelial and tumor cells also take up iron nanoparticles. Larger SPIO particles are identified and rapidly engulfed, principally by resident macrophages in the lymphoreticular organs including the liver, spleen, and lymph nodes. In contrast, USPIO particles are much smaller and therefore less readily recognized by cells, escaping immediate phagocytosis and resulting in a much longer half-life within the blood pool compared to larger particles (up to 36 hours for $\mathrm{USPIO}^{4}$ versus as low as 2 hours for larger $\mathrm{SPIO}^{5}$ in humans). The biodistribution of USPIO is individual to each particle but a blood pool half-life of several hours is expected with distribution predominantly in the spleen, liver, bone marrow, and lymph nodes, before being cleared within a matter of a few days. Cytotoxicity data for iron nanoparticles is limited but is not found until exposure to high levels of iron and is also dependent on factors such as surface coating or its breakdown products, composition of the cell medium, and protein-iron nanoparticle interaction. ${ }^{6-9}$

Whilst circulating within the blood pool, USPIO are small enough to migrate passively across the endothelium ${ }^{1}$ especially in regions of the vasculature where there is loss of endothelial integrity. Once present within tissues, nanoparticles are concentrated and engulfed by resident macrophages through phagocytosis and pinocytosis resulting in their concentration within inflamed tissues. This concentration results in magnetic cooperativity, enhancing their superparamagnetic effects, markedly shortening the $\mathrm{T} 2$ * relaxation time and creating local signal deficits on T2- and $\mathrm{T} 2 *$-weighted images. Thus USPIO can highlight areas of tissue inflammation on MRI. ${ }^{10}$

Earlier studies using iron nanoparticles employed semiquantitative methods to calculate reduction in signal intensity consistent with iron accumulation, however this is liable to variations in signal across the image relative to proximity to surface coils between visits, and also assumes that signal intensity in the control tissue is consistent between both pre- and post-USPIO scans. Calculation of changes in $\mathrm{T} 2$ and $\mathrm{T} 2 *$ relaxivity values has been adopted for a more sensitive and objective approach. The $\mathrm{T} 2$ and $\mathrm{T} 2 *$ relaxivity values, are the initial decay constants for the exponential decay of signal intensity with increasing echo time. Calculation and comparison of decay constants for each region of interest allows for a more objective and quantitative analysis approach and thus accounts for problems caused by variations in relaxivity values between regions and also MRI coil sensitivity between subject visits. In the presence of USPIO the signal decays more rapidly and the $\mathrm{T} 2$ and $\mathrm{T} 2 *$ relaxivity values are reduced. On the resulting images, particularly on $\mathrm{T} 2 *$-weighted images, a profound reduction in signal intensity is evident as a region of signal void (hypointense region) where USPIOs have accumulated (Figure 1).

Precise blood suppression during USPIO-enhanced MRI is important to reduce artifact into nearby myocardium,
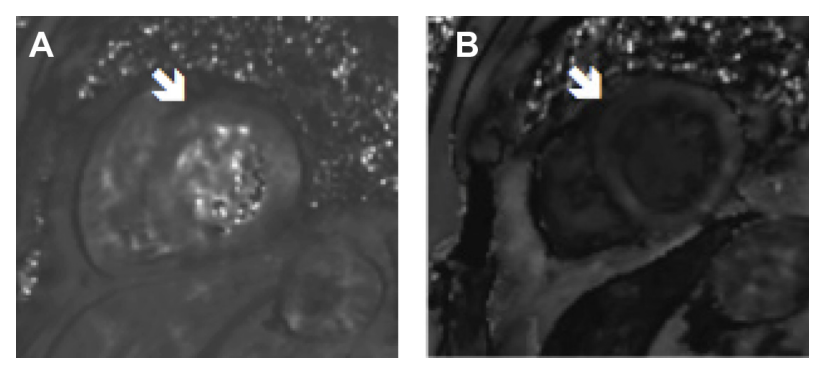

Figure I Patient imaged 3 days after an anterior myocardial infarct with T2*weighted magnetic resonance imaging.

Notes: Images show T2* maps of baseline pre-iron scan (A), and 24 hours following ferumoxytol (USPIO) administration (B). Iron uptake is seen as signal void (dark) in the region of the anterior infarct (arrow).

Abbreviation: USPIO, ultra-small superparamagnetic particles of iron oxide. 
which is often the region of interest under examination. Magnetization transfer between the blood pool and myocardium, and also flow-related blood pool artifact, may compromise the quality of T2* data. Double inversion techniques are commonly used to suppress blood signal in most cardiac MRI examinations. However, double inversion techniques do not account for changes in blood pool USPIO related T1 shortening. Therefore, more complicated inversion strategies (such as a quadrature inversion technique) may be required to fully null blood signal in both pre- and post-USPIO data, although these inversion techniques are less commonly available on clinical systems and so are not widely used.

Here, we focus on the use of USPIO in cardiovascular imaging, discussing their application as a blood pool contrast agent, a tracer highlighting inflammatory cells of the reticuloendothelial system, and in targeted imaging conjugated to a specific antibody or peptide (Table 2).

\section{Cardiac imaging Blood pool contrast}

USPIO have been used successfully as coronary and ventricular MRI blood pool contrast agents for over a decade as they have longer blood pool circulating time compared to standard MRI angiographic agents such as gadolinium. In 1997, Stillman et a ${ }^{11}$ demonstrated improved myocardial to blood pool border definition with ferumoxtran-10 on T1-weighted MRI cine images. Image intensity gradients at the myocardial to blood pool interface increased significantly in both long and short axis views. At the time it was thought that ferumoxtran-10 would improve automated ventricular cavity edge detection.

Table 2 Summary of USPIO studies discussed in this article

\begin{tabular}{|c|c|c|c|c|c|c|c|}
\hline Agent name & $\begin{array}{l}\text { Iron oxide cored } \\
\text { diameter, } \mathrm{nm} \\
\text { (total diameter) }\end{array}$ & Coating & $\begin{array}{l}\text { Magnet } \\
\text { strength }\end{array}$ & $\begin{array}{l}\text { Administration } \\
\text { site }\end{array}$ & $\begin{array}{l}\text { Clinical/ } \\
\text { preclinical }\end{array}$ & Application & Reference \\
\hline \multirow{10}{*}{$\begin{array}{l}\text { Ferumoxtran-10 } \\
\text { (AMI-227, } \\
\text { Combidex }^{\circledR} \text {, } \\
\text { Sinerem }^{\circledR} \text { ) }\end{array}$} & \multirow[t]{10}{*}{$6.3(35)$} & \multirow[t]{10}{*}{ Dextran } & $\mathrm{I} .5 \mathrm{~T}$ & Intravenous & Clinical & Ventricular contrast & 11 \\
\hline & & & $\mathrm{I} .5 \mathrm{~T}$ & Intravenous & Preclinical & $\begin{array}{l}\text { Cardiac transplant } \\
\text { rejection }\end{array}$ & 26,28 \\
\hline & & & $7 \mathrm{~T}$ & Intramyocardial & Preclinical & Stem cells & 36 \\
\hline & & & $\mathrm{I} .5 \mathrm{~T}$ & Intravenous & Preclinical & Atherosclerosis & 50,51 \\
\hline & & & $\mathrm{I} .5 \mathrm{~T}$ & Intravenous & Clinical & Atherosclerosis & $\begin{array}{l}52-55 \\
57-59,62\end{array}$ \\
\hline & & & $1.5 \mathrm{~T}$ & Intravenous & Clinical & Atherosclerosis + AAA & 76 \\
\hline & & & $1.5 \mathrm{~T}$ & Intravenous & Clinical & Aortic + iliac aneurysms & 77 \\
\hline & & & $1.5 \mathrm{~T}$ & Intravenous & Clinical & AAA & 78 \\
\hline & & & $3 \mathrm{~T}$ & Intravenous & Clinical & AAA & 79 \\
\hline & & & $\mathrm{I} .5 \mathrm{~T}$ & Intravenous & Clinical & Lymph node metastases & 80 \\
\hline \multirow{6}{*}{$\begin{array}{l}\text { NCI00I50 } \\
\left.\text { (Clariscan }^{\mathrm{TM}}\right)\end{array}$} & \multirow[t]{6}{*}{$6.43(20)$} & \multirow[t]{6}{*}{ Dextran } & $0.5 / 1.5 \mathrm{~T}$ & Intravenous & Clinical & Ventricular contrast & $12-15$ \\
\hline & & & $1.5 \mathrm{~T}$ & Intravenous & Preclinical & Coronary contrast & 16 \\
\hline & & & $1.5 \mathrm{~T}$ & Intravenous & Clinical & Coronary contrast & 17,18 \\
\hline & & & $1.5 \mathrm{~T}$ & Intravenous & Preclinical & Myocardial contrast & 19,20 \\
\hline & & & $2 \mathrm{~T}$ & Intravenous & Preclinical & Myocardial contrast & 21 \\
\hline & & & $1.5 \mathrm{~T}$ & Intravenous & Preclinical & $\begin{array}{l}\text { Cardiac transplant } \\
\text { rejection }\end{array}$ & 25,26 \\
\hline $\mathrm{n} / \mathrm{a}$ & n/a (27) & Dextran & $4.7 \mathrm{~T}$ & Intravenous & Preclinical & $\begin{array}{l}\text { Cardiac transplant } \\
\text { rejection }\end{array}$ & 24,27 \\
\hline $\mathrm{n} / \mathrm{a}$ & $6(n / a)$ & Polymer & $1.5 \mathrm{~T}$ & Intravenous & Preclinical & $\begin{array}{l}\text { Cardiac allograft } \\
\text { rejection }\end{array}$ & 30 \\
\hline \multirow[t]{5}{*}{ n/a (CLIO) } & \multirow[t]{5}{*}{$3-5(15-20)$} & \multirow[t]{5}{*}{ Dextran } & $9.4 \mathrm{~T}$ & Intravenous & Preclinical & Myocardial infarction & 32 \\
\hline & & & $9.4 \mathrm{~T}$ & Intramyocardial & Preclinical & Stem cells & 37 \\
\hline & & & $1.5 \mathrm{~T}$ & $\begin{array}{l}\text { Gel Phantom/ } \\
\text { intramyocardial }\end{array}$ & $\begin{array}{l}\text { In vitro/ } \\
\text { ex vivo }\end{array}$ & Stem cells & 39 \\
\hline & & & $\mathrm{n} / \mathrm{a}$ & n/a (in-vitro) & In vitro & Apoptosis & 40 \\
\hline & & & $9.4 \mathrm{~T}$ & Intravenous & Preclinical & Apoptosis & 41,42 \\
\hline Ferumoxytol & $3.25(|7-3|)$ & Dextran & $3 \mathrm{~T}$ & Intravenous & Clinical & Myocardial infarction & 33 \\
\hline (Rienso $^{\circledR}$, & & & $1.5 \mathrm{~T}$ & Intravenous & Clinical & Myocardial infarction & 34 \\
\hline \multicolumn{8}{|l|}{ Feraheme ${ }^{\circledR}$ ) } \\
\hline $\mathrm{n} / \mathrm{a}$ & $\mathrm{n} / \mathrm{a}(7.5)$ & Amine silane & $3 \mathrm{~T}$ & Intracoronary & Preclinical & Stem cells & 38 \\
\hline $\mathrm{n} / \mathrm{a}$ & $2-7(15-40)$ & Polysaccharide & $1.5 \mathrm{~T}$ & Intravenous & Preclinical & Atherosclerosis & 49 \\
\hline
\end{tabular}

Note: Rienso ${ }^{\circledR}$; Takeda Italia S.p.A, Rome, Italy.

Abbreviations: USPIO, ultra-small superparamagnetic particles of iron oxide; AAA, abdominal aortic aneurysms; CLIO, cross-linked particles of iron oxide; n/a, not available. 
This was soon followed up by Taylor et $\mathrm{al}^{12}$ who used an alternative USPIO agent, $\mathrm{NC} 100150$ (Clariscan $^{\mathrm{TM}}$ ) with a blood pool half-life of over 2 hours, to aid ventricular cavity edge detection using MRI. They administered NC100150 to 18 healthy male volunteers and examined optimized cine imaging sequences. At higher doses ( 3 and $4 \mathrm{mg} \mathrm{Fe} / \mathrm{kg}$ ) a reduction in signal intensity was seen in the blood pool enabling enhanced detection of the left ventricle blood pool to myocardial interface thus improving functional assessment of the left ventricle. The finding that $\mathrm{NC100150}$ improves myocardial blood pool definition has since been repeated in other human studies. ${ }^{13-15}$

NC100150 has been used extensively as a coronary contrast agent for MRI coronary angiography (MRCA) with mixed results. Johansson et al compared invasive X-ray angiography with T1-weighted MRCA using NC100150 in pigs with coronary artery stenosis. ${ }^{16}$ Although the sample size was small $(n=6)$, the location and severity grading of stenoses on MRCA correlated well with X-ray angiography findings in all animals. Within a few years, Sandstede et a ${ }^{17}$ carried out a feasibility study using the same contrast agent in patients with coronary artery disease. Proximal segments of coronary arteries could be visualized but distal visualization was poor. Furthermore, no improvement in image quality was seen when an increased USPIO dose was used and only six of eight proximal coronary stenoses seen on conventional angiography were detected on the NC100150 contrast enhanced scan.

Klein et $\mathrm{al}^{18}$ conducted an experiment comparing NC100150 enhanced MRI with unenhanced MRI imaging, again yielding mixed results. Although the visible length of mid and distal portions of the coronary artery improved on USPIO-enhanced imaging, there was no improvement when assessing the proximal portion of the artery. Furthermore, only eight of the eleven significant coronary stenoses $(>50 \%)$ were detected on USPIO-enhanced MRI compared to six on the unenhanced scan.

Soon after the development of USPIO as a blood pool contrast agent for ventricular cavity and coronary imaging, attempts were made to illustrate myocardial viability with conflicting results. Kroft et al ${ }^{19}$ administered ferumoxtran-10 (AMI-227, Sinerem $\left.{ }^{\circledR}\right)$ in a pig model of myocardial infarction (MI) and then imaged using T1-weighted MRI with a $1.5 \mathrm{~T}$ scanner. Interim imaging failed to detect USPIO in the viable myocardium, likely due to low dose, fast elimination time of the contrast agent, and a low field strength scanner. Increasing the magnetic field strength provides improved signal-to-noise ratio, greater spatial resolution, and higher sensitivity for detecting lower concentrations of iron, which is advantageous in the detection of USPIO.

Bjerner et $\mathrm{al}^{20}$ had more success using NC100150 in a similar model. Using T1-, T2-, and T2*-weighted imaging, they found that T1-weighted MRI with doses of 4 and $8 \mathrm{mg}$ $\mathrm{Fe} / \mathrm{kg}$ produced the best evaluation of perfused myocardium after histological validation against areas of fluorescein stain uptake on ex vivo histological analysis.

The use of USPIO as a myocardial tissue contrast agent was extended to a model of ischemia-reperfusion in rats to evaluate microvascular injury in reperfused myocardium. Krombach et $\mathrm{al}^{21}$ employed coronary ligation for 45 minutes and 3 hours of reperfusion, and administered NC100150, to help define regions of microvascular injury and obstruction after MI. USPIO distributes by diffusion in the extravascular space of injured myocardium with damaged, leaky vasculature. They found that USPIO-enhanced imaging provided some indication of transmural and non-transmural microvascular injury. This imaging approach tended to overestimate the area of true infarction when compared to triphenyltetrazolium chloride stain, and underestimate the area of myocardium deemed "at risk" of infarction determined by redo coronary ligation and intravenous phthalocyanine blue dye injection.

\section{Inflammation}

Endomyocardial biopsy is the "gold standard" diagnostic investigation for inflammatory diseases of the myocardium. This does carry the potential for life-threatening complication, ${ }^{22}$ and sampling error is common due to the focal nature and spatial heterogeneity of underlying inflammatory processes. This procedure is rarely performed outside specialist tertiary referral centers.

\section{Transplant rejection}

Cardiac transplantation is a life prolonging treatment option for end-stage cardiac disease. Transplant rejection is a major threat to the allograft, occurring in $20 \%-30 \%$ of recipients in the first year. ${ }^{23}$ Rejection can occur at any stage after transplantation and causes significant morbidity and mortality. Serial surveillance myocardial biopsies are routinely undertaken as rejection is notoriously difficult to diagnose using existing non-invasive imaging methods.

Kanno et $\mathrm{al}^{24}$ exploited the finding that iron nanoparticles accumulated within the cytoplasm of macrophages, and detected organ rejection using MRI and dextran-coated USPIO in rats with evidence of cardiac allograft rejection. 
Histopathology confirmed active rejection and identified USPIO uptake confined to macrophages that had accumulated within the rejecting cardiac allograft. Rats that received more prolonged immunosuppression had a decline in the rate of signal intensity reduction, suggesting less inflammation and indicating that treatment response could be monitored with this method.

Subsequent studies have confirmed the value of using USPIO to detect acute cardiac rejection in rats, ${ }^{25-27}$ although ferumoxtran-10 proved inferior to $\mathrm{NC} 100150$ at detecting rejection ${ }^{26}$ and was unable to detect acute cardiac rejection in rats in another study. ${ }^{28}$ In the latter study, ferumoxtran-10 was given at two doses of $2 \mathrm{mg}$ and $11 \mathrm{mg} \mathrm{Fe} / \mathrm{kg}$ with T2* weighted MRI conducted using a $1.5 \mathrm{~T}$ scanner at postoperative day 7, 24 hours after USPIO administration. The failure to detect USPIO was thought to be due to the low field strength magnet of the MRI scanner.

Iron oxide particles can be conjugated to a specific antibody or peptide to target pathophysiological processes. ${ }^{29}$ Recent work by Guo et a $\mathrm{l}^{30}$ targeted $\mathrm{T}$ cells that play a central role in acute cardiac allograft rejection. They built on work performed using ligand targeting of cells to employ a "theranostic" strategy, combining diagnostic imaging with administration of therapy within the one system (Figure 2). They targeted $\mathrm{T}$ cells in the endocardium of rejecting rat hearts using a CD-3 antibody-targeted multifunctional polymeric nanocarrier. This polyplex nanoparticle was further conjugated to a therapeutic gene ( $p D N A-D G K a l p h a$ ) enabling simultaneous imaging and immune modulation of CD-3 expressing $\mathrm{T}$ cells.

\section{$\mathrm{MI}$}

The development of cross-linked particles of iron oxide $(\mathrm{CLIO})^{31}$ allowed targeted cardiac inflammatory imaging. Cross-linking and amination of the USPIO dextran chains encourages circumferential adherence of the dextran coat to the iron nanoparticle providing chemical stability and improved conjugation. ${ }^{31}$ Sosnovik et al ${ }^{32}$ conjugated CLIO with cyanine 5.5 dye creating a "magnetofluorescent" particle allowing co-registration of MRI and fluorescence molecular tomography data. They administered CLIO-Cy5.5 48 hours after left coronary artery ligation in a mouse model of MI with dual modality imaging 48 hours later. The accumulation of CLIO-Cy5.5 within infarcted regions of myocardium was clearly seen on fluorescence and MRI images that were not present on control mice (mice injected with CLIO-Cy5.5 but a "sham" operation). In vivo imaging findings were subsequently confirmed with histology. Examination of infarcted

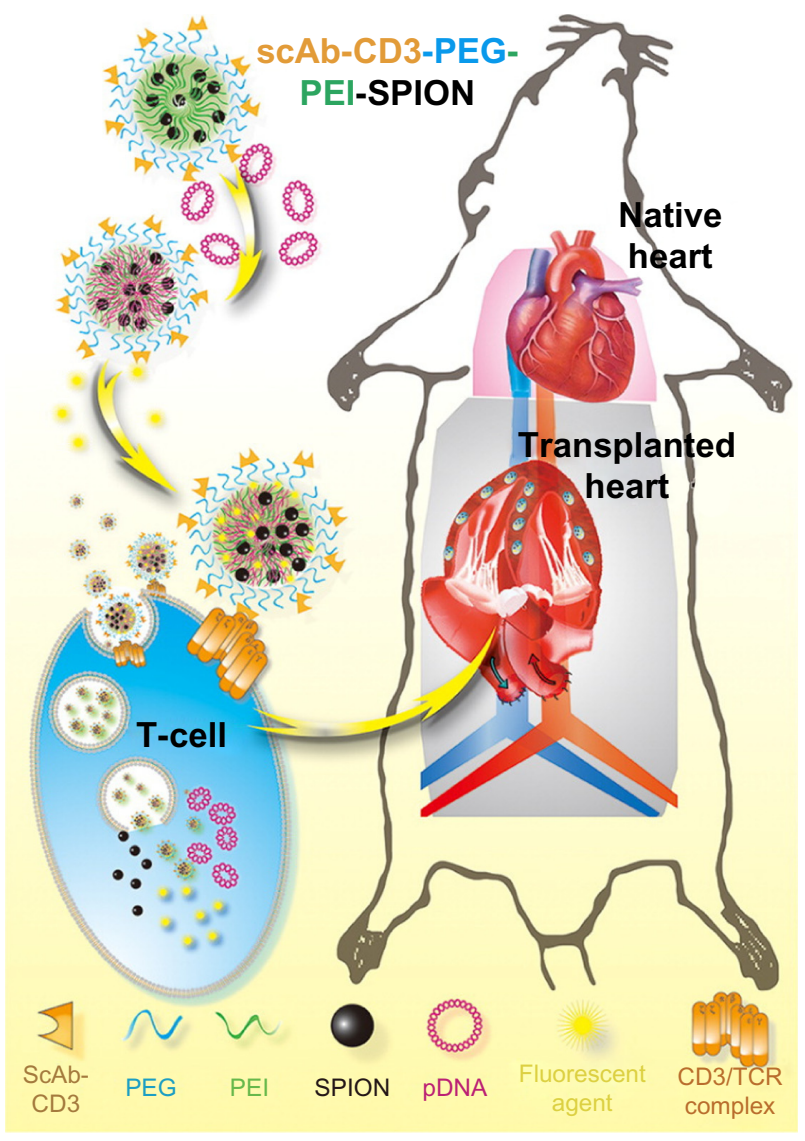

Figure 2 Schematic diagram of therapeutic process of magnetic targeting polyplex scAbCD3-PEG-g-PEI-SPION in vitro and in vivo.

Notes: Reprinted with permission from Guo Y, Chen W, Wang W, et al. Simultaneous diagnosis and gene therapy of immuno-rejection in rat allogeneic heart transplantation model using a T-cell-targeted theranostic nanosystem. ACS Nano. 20I2;6(I2):I0646-10657. Copyright (C) 2012 American Chemical Society. ${ }^{30}$

Abbreviations: ScAb-CD3, CD3 single chain antibody; PEG, polyethylene glycol; PEI, polyethylenimine; SPION, superparamagnetic iron oxide nanoparticles; pDNA, Plasmid DNA; TCR, T-cell receptor.

myocardium revealed a high degree of co-localization between inflammatory cell infiltrates on hematoxylin and eosin staining, increased signal intensity on fluorescence microscopy, and an abundance of macrophages on immunohistochemistry staining. This is consistent with uptake of CLIO-Cy5.5 by macrophages infiltrating the infarcted myocardium.

Clinical applications of USPIO in highlighting myocardial inflammation after MI have since emerged following the discovery that inflammation around the time of MI affects both the extent of myocardial salvage and recovery of left ventricular function. Our group ${ }^{33}$ used USPIO to image myocardial inflammation following acute MI. Within 48 hours following acute MI, patients underwent MRI scanning at baseline (pre-USPIO) before receiving an infusion of ferumoxytol (USPIO) with subsequent serial MRI scans thereafter. The infarct zone was defined by delayed enhancement with gadolinium. Following 
USPIO administration, there was a significant increase in $\mathrm{R} 2 *(\mathrm{R} 2 *=1 / \mathrm{T} 2 *)$ in the infarct zone, with smaller changes observed in the peri-infarct zone (surrounding areas of delayed enhancement) and even in remote myocardium when compared with control tissue (skeletal muscle). This suggested peri-infarct and remote myocardial inflammation, although no histological data were available to confirm this. Notably, the R2* value was greatest 24 hours following USPIO (ferumoxytol) infusion, and had declined by 48 hours suggesting that 24 hours post-infusion is a more sensitive imaging time than 48 hours post-infusion in this group of patients.

Yilmaz et $\mathrm{al}^{34}$ also detected a reduction in $\mathrm{T} 2 *$ value in the infarct as well as in the peri-infarct region, the remote myocardium, and the lymphoreticular system. On imaging the first three patients at 6, 24, 48 and 96 hours after ferumoxytol administration, they attempted to find the optimal time window to characterize inflammation within the myocardium. From these data, they suggest that signal attenuation was maximal at 24 hours but that the best combination of T2-weighted (hypoenhancement seen) and T2*-weighted images was obtained at 48 hours.

Histological data are required to address the outstanding question regarding distribution of USPIO within the myocardium and the relative contribution to changes in $\mathrm{T}_{2} *$ of extracellular iron accumulating within the interstitium, capillary bed, and inflammatory macrophages.

The finding of $\mathrm{T} 2 *$ signal void in remote myocardium suggests a pan-myocardial inflammatory response in the context of a focal infarct. Grieve et $\mathrm{al}^{35}$ confirmed this finding in remote segments in a rat model of ischemia-reperfusion injury examining endovascular inflammation using a targeted larger iron oxide tracer (MPIO).

\section{Other cardiac imaging}

\section{Stem cells}

Current research endeavors are increasingly focusing on stem cell therapy following the discovery that pluripotent progenitor cells bear the capacity to differentiate into mature cardiac cells, in an attempt to restore function to organs with no regenerative capacity. There is a need to track stem cells following administration so that their distribution and fate can be determined. Cell labeling with iron nanoparticles is relatively simple, fast, inexpensive and is well described, although there have been few published studies using USPIO in cardiac stem cell imaging. Tracking of stem cells labeled with iron nanoparticles using MRI is still at the pre-clinical stage but offers high spatial resolution, excellent soft tissue detail, and avoids exposure to ionizing radiation.
Küstermann et $\mathrm{al}^{36}$ labeled cardiac progenitor cells expressing enhanced green fluorescent protein with ferumoxtran-10 and implanted the cells into murine myocardium injured through cryocoagulation or coronary artery ligation. Co-registration of USPIO and stem cells was confirmed by comparing high-resolution MRI images with Prussian Blue stain for iron on histology, and immunohistochemistry for enhanced green fluorescent protein. Difficulties were encountered using this approach as the signal attenuation of unlabeled ischemic myocardium was similar to that induced by USPIO-labeled cells in T2*-weighted images making detection of labeled cells within infarcted regions difficult. However, the authors subsequently found that the combination of proton density- and $\mathrm{T} 2 *$-weighted MRI sequences identified regions of myocardial injury and USPIO-labeled cells respectively - the larger area of signal attenuation in the $\mathrm{T} 2 *$-weighted images was caused by infiltration of the iron-labeled cells.

Chapon et $\mathrm{al}^{37}$ combined MRI and positron emission tomography to track transplanted stem cells after MI in rats providing anatomical, physiological, and metabolic information. They conjugated rat bone marrow-derived stem cells to CLIO and fluorescein dye enabling co-localization of stem cells to be assessed by in vivo MRI and ex vivo magnetic resonance spectroscopy. Stem cells were successfully detected on MRI and histology for the 6 week duration of the study, however positron emission tomography signs of metabolic activity (18F-fluorodeoxyglucose uptake) was only seen in the first week post-stem cell implantation. This may have been due to early, localized inflammation from stem cell injection or more likely death of the stem cells. A trend to an improvement in cardiac function was found after stem cell implantation, although this did not reach statistical significance.

Ma et $\mathrm{al}^{38}$ compared the ability of USPIO and MPIO in tracking stem cells via intracoronary injection, as opposed to a direct intramyocardial route, in a swine model of ischemiareperfusion injury. They found that similar labeling efficiency was found in both particles, and differentiation and proliferation of mesenchymal stem cells (MSCs) in vivo were not affected by labeling with either iron particle, confirmed by immunohistochemical assays showing similar protein expression. Hypointensity was seen in infarcted regions with both particles on T2*-weighted MRI at 4 and 8 weeks and co-localized with regions of iron uptake seen on Prussian Blue staining. However there was no hypointensity detected in 2/7 of the MPIO labeled pigs and the authors suggested that USPIO labeling was more suited to intracoronary injection than the larger MPIO particle. 
Mathiasen et $\mathrm{al}^{39}$ recently sought to determine the MRI detection limits of CLIO labeled human MSCs using gelphantoms in a clinical $1.5 \mathrm{~T}$ MR scanner. They determined the detection limit to be 250,000 MSCs using a 21 hours' incubation time and $10 \mu \mathrm{g}$ USPIO/10 $/ 0^{5}$ MSCs. At lower incubation times and doses of USPIO, significantly more cells were needed for detection. They confirmed their findings in ex vivo porcine myocardium by visualizing the USPIOlabeled MSCs.

USPIO-enhanced MRI appears well placed to provide future clinical research opportunities to detect and track stem cells following implantation. However, to enhance sensitivity, detection limits for stem cell number and administered iron concentration for a particular MRI scanner would need to be determined in advance.

\section{Apoptosis}

During apoptosis, or programmed cell death, the phospholipid phosphatidylserine is externalized on the cell surface. Annexin $\mathrm{V}$ is an endogenous protein that binds with high affinity to phosphatidylserine, and when conjugated to CLIO, has successfully been able to identify apoptotic cells in vitro using MRI. ${ }^{40}$ Sosnovik et al ${ }^{41}$ used this particle as a foundation to create a multimodal nanoparticle further conjugated to cyanine 5.5 dye - AnxCLIO-Cy5.5 - allowing co-identification with fluorescence microscopy and T2*-weighted MRI. In a murine model of ischemia reperfusion injury, a reduction in signal intensity on $\mathrm{T} 2 *$-weighted imaging was observed in reperfused regions with AnxCLIO-Cy5.5 but not with unlabeled CLIO-Cy5.5 suggesting uptake of AnxCLIO-Cy5.5 within the injured and apoptotic myocardium. The area of signal loss correlated with the region of contractile dysfunction seen on MRI, and subsequently with areas of increased fluorescence intensity on near infra-red fluorescence.

More recently, Sosnovik et al employed a similar approach in developing a novel dual-contrast molecular MRI probe capable of detecting cardiomyocyte apoptosis and necrosis in mice with ischemia reperfusion injury in vivo. ${ }^{42}$ Using the same AnxCLIO-Cy5.5 particle to identify cellular apoptosis on MRI 4-6 hours after ischemia-reperfusion injury, they also administered a novel magnetofluorescent gadolinium chelate with subsequent gadolinium delayed enhancement MRI to illustrate necrosis. Interestingly, they found that only $21 \%$ of apoptotic myocardium (accumulation of AnxCLIO-Cy5.5) had evidence of necrosis (defined by delayed gadolinium enhancement) suggesting that the majority of apoptotic cardiomyocytes remain viable 4-6 hours after ischemia-reperfusion injury. This was confirmed on fluores- cence microscopy and immunohistochemistry revealing the presence of large numbers of apoptotic, but potentially viable, cardiomyocytes in the "at-risk" area (AnxCLIO-Cy5.5 positive, Gd-DTPA-NBD negative areas). The authors remarked that strategies to salvage apoptotic, but not yet necrotic, myocardium should be explored now that these areas could be successfully imaged.

\section{Carotid atherosclerosis}

Carotid atherosclerosis and stroke are a major cause of disability and death. In the United States it is estimated that around 750,000 people become victims of stroke annually. ${ }^{43}$ Over one million Americans live with disabilities caused by stroke and $28 \%$ of strokes occur in people under the age of $65 .{ }^{44}$

Although the traditional imaging marker of disease burden has been stenosis, this is a poor marker of disease activity. It is now accepted that atherosclerosis is an inflammatory disorder ${ }^{45-47}$ involving recruitment of inflammatory cells into the lipid-rich plaque core. In atherosclerotic plaques, high macrophage density is a feature of vulnerability and correlates with risk of plaque rupture and clinical events. ${ }^{48}$ Modern techniques are now able to assess the composition and pathobiology of the arterial wall itself, and identification of vulnerable plaques at high risk of rupture is therefore key. USPIO-enhanced MRI is well placed to evaluate inflammation within plaques and will be discussed in this section.

Kresse et $a l^{49}$ first demonstrated USPIO uptake in atherosclerosis in vivo. They administered atherosclerotic rabbits custom-made iron oxide nanoparticles, visualizing these on MRI and confirming histologically with Prussian Blue staining. This study was uncontrolled and no attempt was made at quantitative analysis. Ruehm et $\mathrm{al}^{50}$ soon followed this with a more robust protocol. They administered USPIO to hyperlipidemic atherosclerotic rabbits and performed MRI scans at multiple time points. They noted extensive but patchy signal voids along the length of the aortic wall in atherosclerotic rabbits, but not in controls. Aortae were harvested for histological assessment and electron microscopy. Hyperlipidemic rabbits given USPIO showed extensive iron deposition in the subintima demonstrated by Prussian Blue staining, and on electron microscopy, iron inclusions were noted within plaque macrophages.

Hyafil et al ${ }^{51}$ examined hypercholesterolemic rabbits following iatrogenic aortic injury from balloon angioplasty and conducted MRI sequences at various time points following ferumoxtran-10 administration. At 5 days post-infusion, signal voids were seen in injured aortae on in vivo MRI, extending both inward into the lumen and outward into the periaortic region 
creating a "pseudostenosis" appearance. There was significantly more signal void in balloon-injured aortae compared to control aortae (uninjured aortic regions or rabbits administered saline instead of USPIO). Regions of signal void correlated with iron and RAM-11 staining (a rabbit macrophage marker), and a close correlation was also noted between the distribution of iron and RAM-11 staining. Extensive histological iron staining was seen in the balloon-injured aortae.

The first demonstration of the feasibility of atherosclerosis imaging using USPIO in humans was reported by Schmitz et $\mathrm{al}^{52}$ in a small study of patients with pelvic malignancy undergoing MRI for assessment of lymphatic metastasis using the USPIO ferumoxtran-10, which was at the time licensed for this indication. They retrospectively examined the aortic bifurcation and detected new focal areas of subintimal signal drop out in seven of 20 patients on $\mathrm{T} 2 *$-weighted imaging that had not been present on the pre-USPIO images. The authors speculated that the presumed accumulation of subintimal USPIO was a consequence of dysfunctional endothelium and that this technique might function as an assay of endothelial integrity.

Kooi et $\mathrm{al}^{53}$ performed the first prospective human study evaluating atherosclerosis using USPIO (ferumoxtran-10) in a carotid endarterectomy model. Eleven patients scheduled to undergo carotid surgery following a symptomatic neurovascular event were imaged with T2*-weighted MRI at three time points: pre-USPIO, 24 and 72 hours post-USPIO. Semi-quantitative image analysis was undertaken by calculating the relative signal intensity in quadrants with visible signal changes and comparing to reference tissue (skeletal muscle). Following surgery, carotid plaque was processed for immunohistochemical analysis and electron microscopy. Significant iron staining was noted in nine of ten plaques eligible for analysis, and regions co-localized with CD68 staining for macrophages. Electron microscopy confirmed this, demonstrating abundant USPIOsized intra-cytoplasmic inclusions within macrophages, which on energy-dispersive X-ray analysis were confirmed to be composed of iron. On magnetic resonance analysis, reduction in relative signal intensity was confined to areas of plaque that demonstrated positive histological iron staining. Maximum difference in pre- versus post-USPIO signal intensity occurred at 24 hours, with the 72 hour window failing to reach significance, suggesting that this time point was too late to image USPIO uptake.

Trivedi et $\mathrm{al}^{54}$ conducted a small study of eight patients scheduled for carotid endarterectomy, performing multisequence MRI at various time points (before, 24, 36, 48, and 72 hours after ferumoxtran-10 administration). Only segments of plaque staining positive for iron revealed signal void on MRI which was clearest on qualitative review of the T2*-weighted images at the 36 and 48 hour time points. This finding was replicated in the quantitative analysis (employing relative signal intensity) revealing greatest relative signal loss between 24 and 48 hours. Histological and electron microscopy analysis revealed most plaque sections demonstrated iron staining and that there was co-localization of iron and macrophage staining (MAC387), but there were regions with large populations of macrophages in the absence of iron staining. Electron microscopy confirmed the presence of USPIO-sized particles within macrophages.

In a subsequent study by the same authors, ${ }^{55} 30$ patients awaiting carotid endarterectomy for severe carotid stenosis were imaged before and 36 hours post-USPIO, in line with the findings of their previous work. MRI analysis was similar and more extensive histological validation was undertaken. Areas of reduced relative signal intensity were seen in 24/27 patients (three excluded for excessive artifact) that corresponded to USPIO/macrophage positive histology, and most samples $(23 / 30)$ revealed iron staining within the plaque. There was a significant correlation between the presence of USPIO (based upon change in relative signal intensity) on MRI and iron staining in plaques with focal but not diffuse distribution of signal void. Co-localization of iron staining with CD68 and MAC387 was seen but only modestly correlated (there were areas seen to stain for iron and not macrophages and vice versa), and in some sections there was extracellular iron staining around small neo-vessels, possibly indicating that these immature and leaky vessels may represent an entry port for USPIO.

The authors speculated that possible reasons for the weak correlation between iron and macrophage staining was a lack of sensitivity of Perls' stain for USPIO, delayed kinetics of ferumoxtran-10 in inflammatory tissue, and that macrophage subtypes might have differing uptake kinetics for USPIO. This latter theory has been given some experimental traction recently in a study which has shown that M1 and M2 macrophages take up USPIO at different rates. ${ }^{56}$ M1 polarized (pro-inflammatory/destructive) macrophages appear to take up relatively less USPIO than M2 polarized (anti-inflammatory/pre-healing) macrophages.

In 2006, Tang et al ${ }^{57}$ used a more objective analysis approach by calculating adjusted mean change in relative signal intensity. Instead of investigator selected regions of interest that are prone to bias, the mean change in relative signal intensity was calculated using multiple sample data points and a statistical model accounting for the correlation 
between signal loss in different quadrants within a slice and different slices within a plaque. They recruited 20 patients due to undergo carotid surgery and compared symptomatic to contralateral asymptomatic carotid artery plaques (mean stenosis $77 \%$ versus $46 \%$ respectively). They found that all symptomatic plaques demonstrated signal void and interestingly, 19/20 patients displayed regions of inflammation on the contralateral asymptomatic side. Symptomatic lesions did, however, have a greater percentage of positive quadrants $(72 \%$ versus $51 \%, P=0.007)$, and a significant reduction in adjusted mean relative signal intensity $(10.9 \% \pm 4.3 \%, P=0.02)$ that was not apparent on the contralateral side. They also observed that, as seen in previous studies, several asymptomatic "stable" plaques actually showed enhancement (signal increase) in the fibrous cap. The authors concluded that the results demonstrate the systemic nature of atherosclerotic inflammation and that this technique may aid risk-stratification of asymptomatic plaques in the future.

The notion of systemic inflammation in atherosclerosis was explored further in another study by Tang et al. ${ }^{58}$ Ten patients awaiting coronary artery bypass graft surgery for active coronary disease with incidental asymptomatic carotid stenosis $(>50 \%)$ were compared to ten individuals with similar severity of asymptomatic carotid stenosis but no known coronary artery disease. Plaques from patients awaiting coronary artery bypass graft surgery showed a significant mean signal intensity attenuation of $16.4 \%$ versus an increase (ie, enhancement) of $8.4 \%$ in the truly asymptomatic group making a true difference of $24.9 \%$ (95\% confidence interval $16.7 \%$ to $33.0 \% ; P<0.001$ ) between groups. These data support the hypothesis that vascular inflammation is a systemic phenomenon measurable by USPIO accumulation and that signal enhancement may indicate a more stable plaque phenotype.

The same authors ${ }^{59}$ sought to clarify the relationship between traditional radiological markers of stroke risk and USPIO uptake in 71 individuals with asymptomatic carotid stenosis of varying severity. USPIO uptake on T2*W MRI was compared to luminal stenosis measurements of North American Symptomatic Carotid Endarterectomy Trial $(\text { NASCET) })^{60}$ and European Carotid Surgery Trial (ECST) ${ }^{61}$ methods, and normalized wall index. The group also made an attempt to model the USPIO data with respect to increasing stenosis severity. As with their previous work, no relationship was evident between either measurement of stenosis severity and plaque inflammation, and both are independent risk factors providing valuable and completely different pathological information. To further assess this, a prospective trial examining outcome data in patients stratified by USPIO uptake would be required.

The ATHEROMA (Atorvastatin THerapy: Effects on Reduction Of Macrophage Activity) trial was designed to evaluate the effects of statin therapy on carotid plaque inflammation. ${ }^{62}$ Forty-seven patients with carotid stenosis of greater than $40 \%$ who demonstrated plaque USPIO uptake were randomized to low $(10 \mathrm{mg})$ or high-dose $(80 \mathrm{mg})$ atorvastatin for 12 weeks with USPIO-enhanced MRI at 6 and 12 weeks. Twenty patients completed 12 weeks of treatment in each study arm. The primary end point was met with a significant reduction in USPIO uptake observed in the high-dose treatment arm at both 6 and 12 weeks. No such difference was evident in the low dose arm. Unsurprisingly, beneficial effects were noted on plasma lipid profiles but of greater interest was that microembolic load assessed by transcranial Doppler was significantly reduced. Furthermore, enhancement was seen in the fibrous cap and subintima in the high-dose but not the low-dose cohort, supporting previous observations that such enhancement may be associated with a more stable plaque phenotype. This study represents progress in developing a reliable imaging-based biomarker of atherosclerotic disease activity and inflammation. However, hard clinical outcomes are still lacking. Degnan et $\mathrm{al}^{63}$ performed a retrospective analysis of all participants screened for the ATHEROMA trial $(n=62)$ who received USPIO-enhanced MRI, and recorded the occurrence of stroke, transient ischaemic attack, MI or cardiovascular death during a median follow-up of 4 years. Although those who had events did appear to have greater mean change in relative signal intensity on MRI, there were small absolute differences that did not reach statistical significance $(P=0.07)$. When the population was divided into USPIO-positive or -negative plaque groups, survival curves did not diverge significantly. The authors conclude that their study was not powered to assess outcome but that there were perhaps encouraging signs emerging from the data.

\section{Abdominal aortic aneurysms}

Abdominal aortic aneurysms (AAAs) are defined as focal dilatations of the aorta, where aortic diameter is $>1.5$ times normal. They affect $5 \%$ of men over the age of 65 , and within this group cause $2 \%-3 \%$ of deaths annually. ${ }^{64,65}$ The vast majority of these deaths occur as a result of aortic rupture, which has a mortality rate of $65 \%-85 \%$ even in those reaching hospital alive ${ }^{66,67}$ Currently, aneurysm diameter alone is the sole parameter used to stratify risk, but up to a fifth of ruptured 
AAAs have a diameter less than the $55 \mathrm{~mm}$ intervention threshold and many patients have larger aneurysms without symptoms. ${ }^{68,69}$ The implementation of aneurysm screening has reduced aneurysm-related mortality by up to $53 \%{ }^{64}$ but will increase the number of patients under long-term surveillance. Due to the unpredictability and non-linearity of aneurysm growth, improvements to the prediction of aneurysm rupture are required. Accordingly, there is a shift in focus from anatomical and morphological imaging to novel cellular and molecular techniques that evaluate lesion vulnerability on the basis of inflammatory and biological activity.

A prominent histological feature of AAAs is infiltration of $\mathrm{T}$ and $\mathrm{B}$ lymphocytes, macrophages, neutrophils, and natural killer cells. ${ }^{70-73}$ These inflammatory cells regulate the release of cytokines, resulting in the expression of cell adhesion molecules, protease production, and release of reactive oxygen species that in turn cause degradation of the extracellular matrix through alterations in the balance of matrix metalloproteinases and tissue inhibitors of matrix metalloproteinases. ${ }^{74,75}$ In addition, inflammation and hypoxia lead to new vessel formation and loss of vascular smooth muscle cells, and it is these mechanisms that predispose to aortic dilatation and increased risk of AAA rupture. Inflammation within the aortic wall is often focally distributed and it is these hotspots of pathological activity that have been the focus of much investigation.

Howarth et al were the first to report USPIO uptake in AAA in man. They described simultaneous USPIO uptake within atheroma of both the internal carotid artery and a $56 \mathrm{~mm}$, asymptomatic AAA. ${ }^{76}$ Truijers et al later described USPIO (ferumoxtran-10) uptake in the wall of a small number of asymptomatic AAAs $(n=6) .{ }^{77}$ They found significantly more USPIO-positive quadrants in aneurysm patients than in age-matched controls, and the patient with the largest number of USPIO-positive quadrants had the largest diameter aneurysm although there was a wide variation in USPIO uptake. A subsequent pilot feasibility study by Sadat et al $(n=14)$ quantified USPIO uptake in the AAA wall by using calculated T 2 and T $2 *$ values. ${ }^{78}$ They found significant correlation between imaging sequences pre- and 36 hours post-USPIO infusion, and both imaging methods were able to detect the presence of USPIO uptake.

Our group evaluated the potential for this technique to predict aneurysm expansion. Twenty-nine asymptomatic patients with AAAs underwent baseline MRI scans followed by intravenous administration of USPIO and repeat MRI scans 24-36 hours later. A similar technique was used to assess iron accumulation; the percentage change in $\mathrm{T} 2 *$ value was calculated following USPIO administration, but additionally, a threshold of significance for change was applied. By applying this threshold based on repeatability data for calculation of the T2* value in vascular tissue of subjects not receiving USPIO, we were able to distinguish changes in $\mathrm{T} 2 *$ associated with USPIO accumulation from artifact. All patients demonstrated non-specific peri-luminal USPIO accumulation, likely reflecting USPIO uptake in the fresh, highly cellular component of the thrombus. Thrombus within AAAs is structurally complex and is known to consist of a continuous network of interconnected canaliculi. ${ }^{79}$ This network may support transportation of inflammatory cells from lumen into the thrombus, and further into aortic wall as seen in some patients. Importantly, despite similar aneurysm sizes, it is these patients with mural USPIO uptake who had evidence of aneurysm growth rates three-fold higher (6.6 $\mathrm{mm}$ /year) than patients with no mural USPIO uptake, suggesting that this technique may be used to identify patients at greater risk of aneurysm growth. Aortic tissue was collected from patients undergoing open surgical aneurysm repair and histological analysis confirmed co-localization
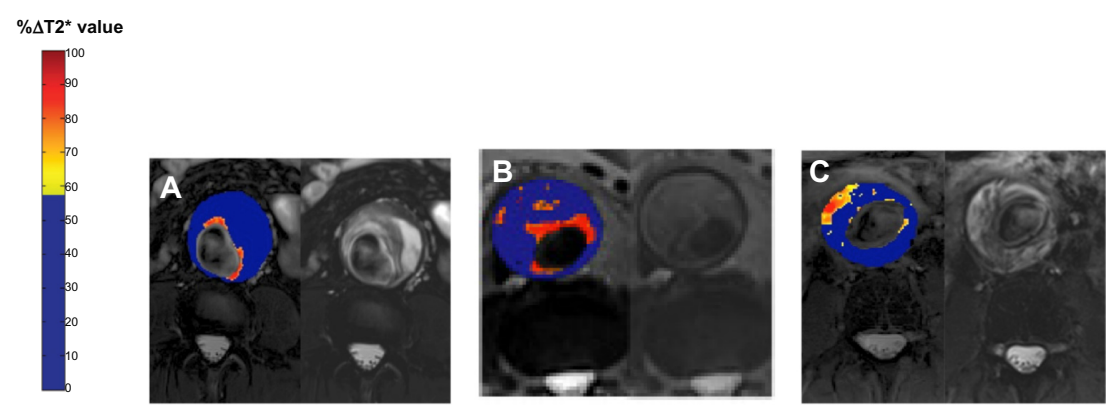

Figure 3 T2*-weighted color maps showing alongside the corresponding T2-weighted anatomic images from patients with abdominal aortic aneurysms (AAA).

Notes: The color scale represents the magnitude of the change in $\mathrm{T}_{2} *$ value, with blue indicating minimal change and red indicating a large change in T2* value using a previously defined threshold above which changes in T2* value can be attributed to ultra-small superparamagnetic particles of iron oxide (USPIO) accumulation. Only changes in $2^{*}$ value above the threshold are presented on the graduated (yellow-red) color scale; data below the threshold appear blue. (A) shows a change in T2* value confined to the peri-luminal area; (B) diffuse patchy changes in T2* throughout the intraluminal thrombus but no distinct focal area of USPIO uptake affecting the aortic wall; and (C) discrete focal area of USPIO uptake involving the wall of the AAA that is distinct from the peri-luminal region. 




Figure 4 Histology from an abdominal aortic aneurysm wall.

Notes: (A) Hematoxylin and eosin staining at $\times 20$ - inflammatory cell infiltrates seen in the endoluminal region with focal calcified atheroma. (B) Perls' stain for iron at $\times 100$ and $(\mathbf{C}) \times 600$ showing the presence of iron (blue). (D) CD68 staining for macrophages (brown) at $\times 100$ and $(\mathbf{E}) \times 600$ showing macrophages colocalizing with iron stain.

of USPIO with CD68 staining for macrophages. ${ }^{80}$ An observational surveillance study is currently underway in our center ( $\mathrm{MA}^{3} \mathrm{RS}$ study) examining whether USPIOenhanced MRI is able to predict the likelihood of rupture or need for surgery (Figures 3 and 4).

\section{Conclusion and future applications}

USPIO can detect inflammation in cardiac, carotid, and aortic disease, whilst providing targeted imaging in stem cell therapy and apoptosis. It is a technique that holds major promise for translation into the clinic.

The development of iron nanoparticle-enhanced MRI has been greatly affected by the discontinuation of several agents in recent years. Ferumoxtran-10 $\left(\right.$ Sinerem $\left.^{\circledR}\right)$, was discontinued due to a high false-positive rate of detecting lymph node metastasis in patients with prostate cancer. ${ }^{81}$ NC100500 (Clariscan ${ }^{\mathrm{TM}}$ ) was discontinued over concerns regarding long-term liver retention, ${ }^{82}$ and the larger SPIO ferumoxides (Feridex ${ }^{\circledR}$ in United States, Endorem ${ }^{\mathrm{TM}}$ in Europe), and ferucarbotran (Resovist ${ }^{\circledR}$ ) have also been discontinued; the latter two agents were the only two clinically approved SPIO agents for MRI. At present there is only one iron oxide particle preparation available for clinical use: ferumoxytol (Rienso $^{\circledR}$; Takeda Italia S.p.A, Rome, Italy), a 17-30 nm USPIO. Rienso is marketed for the intravenous treatment of iron deficiency anemia in adult patients with chronic kidney disease, but is currently not licensed for imaging.

There are several clear opportunities for the developments of USPIO-enhanced MRI as a clinical research tool and our group is using ferumoxytol-enhanced MRI successfully in several clinical research studies. We are attempting to characterize myocardial inflammation following MI in humans: using the assessment of cellular myocardial inflammation as a biomarker for therapeutic interventions. We are also piloting USPIO-enhanced MRI as a diagnostic test in other inflammatory myocardial diseases such as sarcoidosis and myocarditis. At the same time, we are exploring whether USPIO-enhanced MRI can identify patients with high-risk atherosclerotic plaques and AAAs who require early medical or surgical intervention. There are also clear opportunities for USPIO-enhanced MRI in the development of stem cell therapy.

It is difficult to predict where USPIO-enhanced MRI will make a breakthrough into clinical practice but there are clear applications in a range of cardiovascular and noncardiovascular diseases. This exciting imaging technique has great potential and results of research studies in coming years will determine its place in the clinic.

\section{Disclosure}

The British Heart Foundation (FS/07/060, FS/12/83/29781, PG/12/8/29371), National Institutes for Health Research (Efficacy and Mechanism Evaluation, 11/20/03), Medical Research Council (96182) and Chest Heart and Stroke Scotland (r11/A135) and Chief Scientist Office (ETM/266) have provided grants supporting work in this field at our center carried out by CGS, ATV, OMBM, SRA, JMJR, PAH, and DEN. DEN is supported by the British Heart Foundation (CH/09/002). The authors have no other conflicts of interest in this work.

\section{References}

1. Wang XY, Hussain SM, Krestin GP. Superparamagnetic iron oxide contrast agents: physicochemical characteristics and applications in MR imaging. Eur Radiol. 2001;11(11):2319-2331.

2. Allkemper T, Bremer C, Matuszewski L, Ebert W, Reimer P. Contrastenhanced blood-pool MR angiography with optimized iron oxides: effect of size and dose on vascular contrast enhancement in rabbits. Radiology. 2002;223(2):432-438. 
3. Mandeville JB, Moore J, Chesler DA, Garrido L, Weissleder R, Weisskoff RM. Dynamic liver imaging with iron oxide agents: effects of size and biodistribution on contrast. Magn Reson Med. 1997;37(6):885-890.

4. McLachlan SJ, Morris MR, Lucas MA, et al. Phase I clinical evaluation of a new iron oxide MR contrast agent. J Magn Reson Imaging. 1994;4(3):301-307.

5. Clément $\mathrm{O}$, Siauve N, Cuénod CA, Frija G. Liver imaging with ferumoxides (Feridex): fundamentals, controversies, and practical aspects. Top Magn Reson Imaging. 1998;9(3):167-182.

6. Mahmoudi M, Simchi A, Imani M, Milani AS, Stroeve P. An in vitro study of bare and poly(ethylene glycol)-co-fumarate-coated superparamagnetic iron oxide nanoparticles: a new toxicity identification procedure. Nanotechnology. 2009;20(22):225104.

7. Mahmoudi M, Simchi A, Imani M, et al. A new approach for the in vitro identification of the cytotoxicity of superparamagnetic iron oxide nanoparticles. Colloids Surf B Biointerfaces. 2010;75(1):300-309.

8. Berry CC, Wells S, Charles S, Aitchison G, Curtis A. Cell response to dextran-derivatised iron oxide nanoparticles post internalisation. Biomaterials. 2004;25(23):5405-5413.

9. Berry CC, Wells S, Charles S, Curtis AS. Dextran and albumin derivatised iron oxide nanoparticles: influence on fibroblasts in vitro. Biomaterials. 2003;24(25):4551-4557.

10. Wang YX. Superparamagnetic iron oxide based MRI contrast agents: Current status of clinical application. Quant Imaging Med Surg. 2011;1(1):35-40.

11. Stillman AE, Wilke N, Jerosch-Herold M. Use of an intravascular T1 contrast agent to improve MR cine myocardial-blood pool definition in man. J Magn Reson Imaging. 1997;7(4):765-767.

12. Taylor AM, Panting JR, Keegan J, et al. Use of the intravascular contrast agent NC100150 injection in spin-echo and gradient-echo imaging of the heart. J Cardiovasc Magn Reson. 1999;1(1):23-32.

13. Paetsch I, Thiele H, Schnackenburg B, et al. Improved functional cardiac MR imaging using the intravascular contrast agent CLARISCAN. Int J Cardiovasc Imaging. 2003;19(4):337-343.

14. Bunce NH, Moon JC, Bellenger NG, et al. Improved cine cardiovascular magnetic resonance using Clariscan (NC100150 injection). J Cardiovasc Magn Reson. 2001;3(4):303-310.

15. Amano Y, Herfkens RJ, Shifrin RY, Alley MT, Pelc NJ. Threedimensional cardiac cine magnetic resonance imaging with an ultrasmall superparamagnetic iron oxide blood pool agent (NC100150). J Magn Reson Imaging. 2000;11(2):81-86.

16. Johansson LO, Nolan MM, Taniuchi M, Fischer SE, Wickline SA, Lorenz $\mathrm{CH}$. High-resolution magnetic resonance coronary angiography of the entire heart using a new blood-pool agent, NC100150 injection: comparison with invasive $\mathrm{x}$-ray angiography in pigs. J Cardiovasc Magn Reson. 1999;1(2):139-143.

17. Sandstede JJ, Pabst T, Wacker C, et al. Breath-hold 3D MR coronary angiography with a new intravascular contrast agent (feruglose) - first clinical experiences. Magn Reson Imaging. 2001;19(2):201-205.

18. Klein C, Schalla S, Schnackenburg B, et al. Improvement of image quality of non-invasive coronary artery imaging with magnetic resonance by the use of the intravascular contrast agent Clariscan (NC100150 injection) in patients with coronary artery disease. J Magn Reson Imaging. 2003;17(6):656-662.

19. Kroft LJM, Doornbos J, van der Geest RJ, van der Laarse A, van der Meulen H, de Roos A. Ultrasmall superparamagnetic particles of iron oxide (USPIO) MR imaging of infarcted myocardium in pigs. Magn Reson Imaging. 1998;16(7):755-763.

20. Bjerner T, Ericsson A, Wikström G, et al. Evaluation of nonperfused myocardial ischemia with MRI and an intravascular USPIO contrast agent in an ex vivo pig model. J Magn Reson Imaging. 2000;12(6):866-872.

21. Krombach GA, Wendland MF, Higgins CB, Saeed M. MR imaging of spatial extent of microvascular injury in reperfused ischemically injured rat myocardium: value of blood pool ultrasmall superparamagnetic particles of iron oxide. Radiology. 2002;225(2):479-486.
22. Cooper LT, Baughman KL, Feldman AM, et al. The role of endomyocardial biopsy in the management of cardiovascular disease A scientific statement from the American Heart Association, the American College of Cardiology, and the European Society of Cardiology endorsed by the Heart Failure Society of America and the Heart Failure Association of the European Society of Cardiology. J Am Coll Cardiol. 2007;50(19):1914-1931.

23. Hertz MI, Aurora P, Christie JD, et al. Scientific registry of the International Society for Heart and Lung Transplantation: introduction to the 2009 annual reports. $J$ Heart Lung Transplant. 2009;28(10): 989-992.

24. Kanno S, Wu YJ, Lee PC, Dodd SJ, et al. Macrophage accumulation associated with rat cardiac allograft rejection detected by magnetic resonance imaging with ultrasmall superparamagnetic iron oxide particles. Circulation. 2001;104(8):934-938.

25. Johansson L, Johnsson C, Penno E, Bjornerud A, Ahlstrom H. Acute cardiac transplant rejection: detection and grading with MR imaging with a blood pool contrast agent experimental study in the rat. Radiology. 2002;225(1):97-103.

26. Penno E, Johnsson C, Johansson L, Ahlström H. Comparison of ultrasmall superparamagnetic iron oxide particles and low molecular weight contrast agents to detect rejecting transplanted hearts with magnetic resonance imaging. Invest Radiol. 2005;40(10): 648-654.

27. Wu YL, Ye Q, Sato K, Foley LM, Hitchens TK, Ho C. Noninvasive evaluation of cardiac allograft rejection by cellular and functional cardiac magnetic resonance. JACC Cardiovasc Imaging. 2009;2(6):731-741.

28. Penno E, Johnsson C, Johansson L, Ahlstrom H. Macrophage uptake of ultra-small iron oxide particles for magnetic resonance imaging in experimental acute cardiac transplant rejection. Acta Radiol. 2009;47(3):264-271.

29. McAteer MA, Akhtar AM, Zur Muhlen von C, Choudhury RP. An approach to molecular imaging of atherosclerosis, thrombosis, and vascular inflammation using microparticles of iron oxide. Atherosclerosis. 2010;209(1):18-27.

30. Guo Y, Chen W, Wang W, et al. Simultaneous diagnosis and gene therapy of immuno-rejection in rat allogeneic heart transplantation model using a T-cell-targeted theranostic nanosystem. ACS Nano. 2012;6(12):10646-10657.

31. Wunderbaldinger P, Josephson L, Weissleder R. Crosslinked iron oxides (CLIO): a new platform for the development of targeted MR contrast agents. Acad Radiol. 2002;9 Suppl 2:S304-S306.

32. Sosnovik DE, Nahrendorf M, Deliolanis N, et al. Fluorescence tomography and magnetic resonance imaging of myocardial macrophage infiltration in infarcted myocardium in vivo. Circulation. 2007;115(11):1384-1391.

33. Alam SR, Shah AS, Richards J, et al. Ultrasmall superparamagnetic particles of iron oxide in patients with acute myocardial infarction: early clinical experience. Circ Cardiovasc Imaging. 2012;5(5):559-565.

34. Yilmaz A, Dengler MA, van der Kuip H, et al. Imaging of myocardial infarction using ultrasmall superparamagnetic iron oxide nanoparticles: a human study using a multi-parametric cardiovascular magnetic resonance imaging approach. Eur Heart J. 2013;34(6): 462-475.

35. Grieve SM, Lønborg J, Mazhar J, et al. Cardiac magnetic resonance imaging of rapid VCAM-1 up-regulation in myocardial ischemiareperfusion injury. Eur Biophys J. 2012;42(1):61-70.

36. Küstermann E, Roell W, Breitbach M, et al. Stem cell implantation in ischemic mouse heart: a high-resolution magnetic resonance imaging investigation. NMR Biomed. 2005;18(6):362-370.

37. Chapon C, Jackson JS, Aboagye EO, Herlihy AH, Jones WA, Bhakoo KK. An in vivo multimodal imaging study using MRI and PET of stem cell transplantation after myocardial infarction in rats. Mol Imaging Biol. 2009;11(1):31-38.

38. Ma GS, Qi CM, Liu NF, et al. Efficiently tracking of stem cells in vivo using different kinds of superparamagnetic iron oxide in swine with myocardial infarction. Chin Med J (Engl). 2011;124(8): 1199-1204. 
39. Mathiasen AB, Hansen L, Friis T, Thomsen C, Bhakoo K, Kastrup J. Optimal labeling dose, labeling time, and magnetic resonance imaging detection limits of ultrasmall superparamagnetic ironoxide nanoparticle labeled mesenchymal stromal cells. Stem Cells Int. 2013;2013:353105.

40. Schellenberger EA, Högemann D, Josephson L, Weissleder R. Annexin V-CLIO: a nanoparticle for detecting apoptosis by MRI. Acad Radiol. 2002;9 Supp1 2:S310-S311.

41. Sosnovik DE, Schellenberger EA, Nahrendorf M, et al. Magnetic resonance imaging of cardiomyocyte apoptosis with a novel magneto-optical nanoparticle. Magn Reson Med. 2005;54(3): 718-724.

42. Sosnovik DE, Garanger E, Aikawa E, et al. Molecular MRI of cardiomyocyte apoptosis with simultaneous delayed-enhancemen MRI distinguishes apoptotic and necrotic myocytes in vivo potential for midmyocardial salvage in acute ischemia. Circ Cardiovasc Imaging. 2009;2(6):460-467.

43. Sobieszczyk P, Beckman J. Carotid artery disease. Circulation. 2006;114(7):e244-e247.

44. Stroke [webpage on the Internet]. Fairfax: Society of Interventional Radiology; 2014. Available from: http://www.sirweb.org/patients/ stroke/. Accessed August 24, 2014.

45. Ross R. Atherosclerosis - An inflammatory disease. $N$ Engl J Med. 1999;340(2):115-126.

46. Libby P. Inflammation in atherosclerosis. Nature. 2002;420(6917) 868-874.

47. Libby P. Inflammation in Atherosclerosis. Arterioscler Thromb Vasc Biol. 2012;32(9):2045-2051.

48. Naghavi M, Libby P, Falk E, et al. From vulnerable plaque to vulnerable patient: a call for new definitions and risk assessment strategies: part II. Circulation. 2003;108(15):1772-1778.

49. Kresse M, Wagner S, Thode K, Dinkelborg L. MR plaque imaging using superparamagnetic iron oxide particles. Proc ISMRM. 1998.

50. Ruehm SG, Corot C, Vogt P, Kolb S, Debatin JF. Magnetic resonance imaging of atherosclerotic plaque with ultrasmall superparamagnetic particles of iron oxide in hyperlipidemic rabbits. Circulation. 2001;103(3):415-422.

51. Hyafil F, Laissy JP, Mazighi M, et al. Ferumoxtran-10-enhanced MRI of the hypercholesterolemic rabbit aorta: relationship between signal loss and macrophage infiltration. Arterioscler Thromb Vasc Biol. 2006;26(1):176-181.

52. Schmitz SA, Taupitz M, Wagner S, Wolf KJ, Beyersdorff D, Hamm B. Magnetic resonance imaging of atherosclerotic plaques using superparamagnetic iron oxide particles. J Magn Reson Imaging. 2001 14(4):355-361.

53. Kooi ME, Cappendijk VC, Cleutjens KB, et al. Accumulation of ultrasmall superparamagnetic particles of iron oxide in human atherosclerotic plaques can be detected by in vivo magnetic resonance imaging. Circulation. 2003;107(19): 2453-2458.

54. Trivedi RA, U-King-Im JM, Graves MJ, et al. In vivo detection of macrophages in human carotid atheroma: temporal dependence of ultrasmall superparamagnetic particles of iron oxide-enhanced MRI Stroke. 2004;35(7):1631-1635.

55. Trivedi RA, Mallawarachi C, U-King-Im JM, Graves MJ, et al. Identifying inflamed carotid plaques using in vivo USPIO-enhanced MR imaging to label plaque macrophages. Arterioscler Thromb Vasc Biol. 2006;26(7):1601-1606.

56. Satomi T, Ogawa M, Mori I, et al. Comparison of contrast agents for atherosclerosis imaging using cultured macrophages: FDG versus ultrasmall superparamagnetic iron oxide. J Nucl Med. 2013;54(6): 999-1004.

57. Tang T, Howarth SP, Miller SR, et al. Assessment of inflammatory burden contralateral to the symptomatic carotid stenosis using highresolution ultrasmall, superparamagnetic iron oxide-enhanced MRI. Stroke. 2006;37(9):2266-2270.
58. Tang TY, Howarth SP, Miller SR, et al. Comparison of the inflammatory burden of truly asymptomatic carotid atheroma with atherosclerotic plaques in patients with asymptomatic carotid stenosis undergoing coronary artery bypass grafting: an ultrasmall superparamagnetic iron oxide enhanced magnetic resonance study. Eur J Vasc Endovasc Surg. 2008;35(4):392-398.

59. Tang TY, Howarth SP, Miller SR, et al. Correlation of carotid atheromatous plaque inflammation using USPIO-enhanced MR imaging with degree of luminal stenosis. Stroke. 2008;39(7):2144-2147.

60. North American Symptomatic Carotid Endarterectomy Trial Collaborators. Beneficial effect of carotid endarterectomy in symptomatic patients with high-grade carotid stenosis. N Engl J Med. 1991;325(7): $445-453$.

61. [No authors listed] Randomised trial of endarterectomy for recently symptomatic carotid stenosis: final results of the MRC European Carotid Surgery Trial (ECST). Lancet. 1998;351(9113):1379-1387.

62. Tang TY, Howarth SP, Miller SR, et al. The ATHEROMA (Atorvastatin Therapy: Effects on Reduction of Macrophage Activity) study evaluation using ultrasmall superparamagnetic iron oxide-enhanced magnetic resonance imaging in carotid disease. J Am Coll Cardio. 2009;53(22):2039-2050.

63. Degnan AJ, Patterson AJ, Tang TY, Howarth SPS, Gillard JH. Evaluation of ultrasmall superparamagnetic iron oxide-enhanced MRI of carotid atherosclerosis to assess risk of cerebrovascular and cardiovascular events: follow-up of the ATHEROMA trial. Cerebrovasc Dis. 2012;34(2):169-173.

64. Scott R. The Multicentre Aneurysm Screening Study (MASS) into the effect of abdominal aortic aneurysm screening on mortality in men a randomised controlled trial. Lancet. 2002;360(9345):1531-1539.

65. Gillum RF. Epidemiology of aortic aneurysm in the United States. 1995;48(11):1289-1298.

66. Basnyat PS, Biffin AHB, Moseley LG, Hedges AR, Lewis MH. Mortality from ruptured abdominal aortic aneurysm in Wales. British J of Surgery. 1999;86:765-770.

67. Johnston KW. Ruptured abdominal aortic aneurysm: six-year follow-up results of a multicenter prospective study. Canadian Society for Vascular Surgery Aneurysm Study Group. J Vasc Surg. 1994;19(5): 888-900.

68. Darling RC, Messina CR, Brewster DC, Ottinger LW. Autopsy study of unoperated abdominal aortic aneurysms. The case for early resection. Circulation. 1977;56(3 Suppl):II161-II164.

69. Powell JT, Brown LC, Greenhalgh RM, Thompson SG. The rupture rate of large abdominal aortic aneurysms: is this modified by anatomical suitability for endovascular repair? Ann Surg. 2008;247(1): 173-179.

70. Eliason JL, Hannawa KK, Ailawadi G, et al. Neutrophil depletion inhibits experimental abdominal aortic aneurysm formation. Circulation. $2005 ; 112(2): 232-240$

71. Reeps C, Pelisek J, Seidl S, et al. Inflammatory infiltrates and neovessels are relevant sources of MMPs in abdominal aortic aneurysm wall. Pathobiology. 2009;76(5):243-252.

72. Houard X, Ollivier V, Louedec L, Michel JB, Back M. Differential inflammatory activity across human abdominal aortic aneurysms reveals neutrophil-derived leukotriene B4 as a major chemotactic factor released from the intraluminal thrombus. FASEB J. 2009;23(5): 1376-1383.

73. Ocana E, Bohórquez JC, Pérez-Requena J, Brieva JA, Rodríguez C. Characterisation of $\mathrm{T}$ and $\mathrm{B}$ lymphocytes infiltrating abdominal aortic aneurysms. Atherosclerosis. 2003;170(1):39-48.

74. Thompson MM, Wills A, McDermott E, Crowther M, Brindle N, Bell PR. An in vitro model of aneurysmal disease: effect of leukocyte infiltration and shear stress on MMP production within the arterial wall. Ann NY Acad Sci. 1996;800:270-273.

75. Anidjar S, Salzmann JL, Gentric D, Lagneau P, Camilleri JP, Michel JB Elastase-induced experimental aneurysms in rats. Circulation. 1990;82(3):973-981. 
76. Howarth SP, Tang TY, Graves MJ, et al. Non-invasive MR imaging of inflammation in a patient with both asymptomatic carotid atheroma and an abdominal aortic aneurysm: a case report. Ann Surg Innov Res. 2007;1:4.

77. Truijers M, Fütterer JJ, Takahashi S, Heesakkers RA, Blankensteijn JD, Barentsz JO. In vivo imaging of the aneurysm wall with MRI and a macrophage-specific contrast agent. AJR Roentgenol. 2009;193(5): W437-W441.

78. Sadat U, Taviani V, Patterson AJ, et al. Ultrasmall superparamagnetic iron oxide-enhanced magnetic resonance imaging of abdominal aortic aneurysms - A feasibility study. Eur J Vasc Endovasc Surg. 2011;41(2):167-174.
79. Adolph R, Vorp DA, Steed DL, Webster MW, Kameneva MV, Watkins SC. Cellular content and permeability of intraluminal thrombus in abdominal aortic aneurysm. J Vasc Surg. 1997;25(5):916-926.

80. Richards JM, Semple SI, MacGillivray TJ, et al. Abdominal aortic aneurysm growth predicted by uptake of ultrasmall superparamagnetic particles of iron oxide: a pilot study. Circ Cardiovasc Imaging. 2011;4(3):274-281.

81. Heesakkers RA, Jager GJ, Hövels AM, et al. Prostate cancer: detection of lymph node metastases outside the routine surgical area with ferumoxtran-10-enhanced MR imaging. Radiology. 2009;251(2):408-414.

82. Ho V, Reddy GP. Cardiovascular Imaging. Elsevier Health Sciences; 2010.

\section{Publish your work in this journal}

Journal of Vascular Diagnostics is an international, peer-reviewed journal of diagnostics, focusing on non invasive vascular investigation methods involved in the evaluation of vascular diseases. The journal is committed to the rapid publication in the fields of vascular diseases. Original research, review, case reports, expert opinion and commentaries

\section{Dovepress}

are all considered for publication. The manuscript management system is completely online and includes a very quick and fair peer-review system, which is all easy to use. Visit http://www.dovepress.com/testimonials.php to read real quotes from published authors. 